\title{
Prediction of endometrial carcinoma by subjective endometrial intraepithelial neoplasia diagnosis
}

\author{
Jonathan L Hecht ${ }^{1}$, Tan A Ince ${ }^{2}$, Jan PA Baak ${ }^{3}$, Heather E Baker ${ }^{2}$, Maryann W Ogden ${ }^{2}$ and \\ George L Mutter ${ }^{2}$ \\ ${ }^{1}$ Department of Pathology, Beth Israel Hospital, Boston, MA, USA; ${ }^{2}$ Department of Pathology, Brigham and \\ Women's Hospital, Boston, MA, USA and ${ }^{3}$ Department of Pathology, Central Hospital of Rogaland, Stavanger, \\ Norway
}

\begin{abstract}
Endometrial intraepithelial neoplasia (also known as 'EIN') is a precursor to endometrioid endometrial adenocarcinoma characterized by monoclonal growth of mutated cells, a distinctive histopathologic appearance, and 45 -fold elevated cancer risk. We have applied diagnostic criteria for EIN to 97 successive endometrial biopsies classified as hyperplastic according to World Health Organization criteria and correlated results with computer-assisted morphometry ( $D$-score) and clinical cancer outcomes. Three pathologists separately reviewed all cases for presence or absence of EIN using published criteria (gland area $>$ stromal area, cytologic change in focus of altered architecture, lesion size $>1 \mathrm{~mm}$, and exclusion of cancer and mimics). Discordant cases were resolved by a consensus review at a multiheaded scope. Clinical outcomes were obtained in $\mathbf{8 4}$ patients from patient visit and pathology records. Diagnoses of presence or absence of EIN were unanimous among all three pathologists in $75 \%$ of cases, and intraobserver-reproducibility was very good (kappa 0.73-0.90). Cases rediagnosed as EIN encompassed hyperplasias previously diagnosed as atypical $(n=18)$ or nonatypical (eight complex, two simple). Eight follow-up cancers were scattered between hyperplasia types (5/21 atypical, $3 / 63$ nonatypical), but all classified as EIN (8/25) and $D$-score $\leq 1(8 / 38)$. Subjective application of criteria for diagnosis of EIN correlates well with objective morphometry and successfully segregates patients into high and low cancer risk subgroups with better reproducibility than atypical hyperplasia diagnosis.

Modern Pathology (2005) 18, 324-330, advance online publication, 5 November 2004; doi:10.1038/modpathol.3800328
\end{abstract}

Keywords: EIN; endometrial hyperplasia; histomorphometry; precancer; pathology; prognosis

Diagnosis and therapeutic ablation of premalignant lesions of the endometrium is central to endometrial cancer prevention. Accurate diagnosis of precursors to endometrioid endometrial cancer (Type I), ${ }^{1,2}$ which may precede cancer by several years, presents a major challenge to pathologists. The World Health Organization (WHO) endometrial hyperplasia schema captures many precancers in the atypical hyperplasia subgroup, but is a poorly reproducible system. This report examines clinical performance of the alternative EIN diagnostic schema as practiced subjectively at our institution (BWH) since 2002.

Correspondence: Dr GL Mutter, MD, Division of Women's and Perinatal Pathology, Department of Pathology, Brigham and Women's Hospital, 75 Francis Street, Boston, MA 02115, USA. E-mail: gmutter@rics.bwh.harvard.edu

Received 19 August 2004; revised and accepted 24 September 2004; published online 5 November 2004
The endometrial intraepithelial neoplasia (EIN) diagnostic schema ${ }^{3,4}$ is the end product of combined molecular, ${ }^{5,6}$ objective histomorphometric, ${ }^{7}$ and clinical outcome studies ${ }^{8-11}$ specifically intended to redefine the histopathologic appearance of highrisk endometrial lesions. Premalignant lesions arise as clonal outgrowths of somatically mutated cells that present histologically as an expanding discrete focus of crowded glands with a distinctly different cytology than the background endometrium. The EIN schema more precisely defines 'atypia' and gland crowding than previous criteria for hyperplasia. The absolute cytology of EIN lesions varies greatly between patients, and in a number of cases lacks prominent nucleoli or nuclear rounding, definitions of 'atypia' classically associated with atypical hyperplasia. In contrast to a fixed image of nuclear atypia that does not consider the background setting, EIN cytology always differs from the background from which it has emerged. 
Architectural features of EIN have been extrapolated from large follow-up studies of patients whose endometrial biopsies have a $D$-score $\leq 1$ on objective histomorphometric evaluation. This morphometric group of patients has a 45 -fold elevated risk of future endometrial carcinoma compared to those with a $D$ score $>1 .^{7-10,12}$ Key elements of the $D$-score that correspond to heightened risk of cancer outcome include a lesion size exceeding $1 \mathrm{~mm}$ in maximum dimension and a surface area of glands that exceeds that of stroma.

By examining training biopsies known to contain EIN precursor lesions based on morphometry and genetics, we have previously developed subjective criteria for EIN diagnosis, ${ }^{4,13,14}$ which may be implemented in routine practice of hematoxylin and eosin (H\&E) light microscopy without computerassisted formal morphometry or specialized equipment. Here we evaluate its diagnostic and outcome predictive performance. Subjective EIN diagnosis is contrasted with classification of the same cases by objective morphometry ( $D$-score) and the WHO hyperplasia schema. Our expectation was that subjective EIN diagnosis would correlate positively with the $D$-score from which it is derived, yet diverge materially from the WHO hyperplasia schema that fails to evaluate many of those features now known to best predict clinical outcome. These include lesion size, quantitative extent of gland crowding, and use of internal reference points for cytology interpretation.

\section{Materials and methods}

\section{Case Selection}

A total of 102 sequential endometrial biopsies and curettings accessioned between 20 February 1998 and 21 December 2000 at the Beth Israel Hospital Department of Pathology were identified by pathology report review that listed a diagnosis of endometrial hyperplasia without carcinoma. The primary diagnosis was recorded according to the WHO hyperplasia terminology $y^{4,15}$ as used in the original report, including descriptions of both architecture (complex or simple) and cytology (atypical or nonatypical). Routine H\&E-stained file slides were reviewed to select the most representative section. Specimens having coexisting endometrial adenocarcinoma on review $(n=5)$ were excluded. This left 97 cases for the study.

Follow-up was determined from medical record review of clinical visits and results of subsequent endometrial sampling. Clinical outcome was recorded for 84 patients having any follow-up endometrial pathology specimen, or those with a minimum of 1 year of clinical follow-up visits.

\section{Pathologist Review using EIN Criteria}

Slides were diagnosed according to EIN terminology by three gynecologic pathologists (JH, GM, TI) using published criteria. ${ }^{4,16}$ Each pathologist independently reviewed slides on two occasions, after which a consensus adjudication review was performed at a multiheaded microscope by all three pathologists on those cases where the primary diagnoses did not match in at least five of six diagnostic 'passes'.

Areas diagnosed as EIN were required to meet four criteria: (1) area of glands exceeds area of stroma; (2) when a localizing lesion is present, epithelial cells within the architecturally crowded focus was cytologically different compared to background; (3) area meeting architectural and cytologic criteria must have a minimum size of $1 \mathrm{~mm}$; (4) exclusion of mimics and carcinoma. When EIN does occupy the entire endometrial compartment, or a discrete localizing lesion is absent, internal comparisons of cytology between lesion and background are not possible. In our experience, this occurs in only about $25 \%$ of EIN examples, leaving the majority suited for use of a relative internal standard for interpretation of cytology.

Endometria diagnosed as anovulatory had proliferative glands with focal cystic dilatation or branching, with or without associated vascular thrombi and stromal breakdown. Endometrial polyps are localizing lesions that met at least two of the following three diagnostic criteria in an area confirmed to be endometrial functionalis; (1) irregular gland architecture, (2) altered stroma; (3) thick-walled vessels. Carcinoma was diagnosed when one of the following features was present in neoplastic epithelium: (1) 'rambling' or mazelike glands; (2) solid areas of epithelium; (3) significant cribriforming; or (4) threadlike intervening fibrous tissue with polygonal distortion of contiguous glands ('mosaic pattern'). Multiple diagnoses (primary and secondary) were recorded when appropriate.

\section{Computerized Morphometry}

Computerized morphometric analysis of representative delineated regions on $\mathrm{H} \& \mathrm{E}$-stained sections from 95 cases (two were unavailable due to return of slides to the source institution) was performed with the QProdit 6.1 system (Leica, Cambridge, UK) as previously ${ }^{7,9}$ described. For each lesion the $D$-score was calculated, incorporating volume percentage stroma (VPS), standard deviation of shortest nuclear axis (SDSNA), and gland outer surface density (OUTSD), and then classified as probable precancer (EIN) $(D \leq 1, n=42)$, or probable benign $(D>1$, $n=53)$ based on the previously developed outcome-predictive formula $D=0.6229+(0.0439 \mathrm{VPS})-$ (3.9934 Ln(SDSNA)) - (0.1592OUTSD).

\section{Results}

In total, 97 endometrial biopsies were diagnosed using EIN criteria. The first pass diagnoses of the 
three pathologists were concordant with respect to EIN vs non-EIN in $75 \%$ (73/97) of cases. Interobserver reproducibility among the three pathologists was good, with pairwise (between pathologist) kappas of $0.62,0.56,0.54$. Overall intraobserver reproducibility between the two diagnostic passes was $92.8 \%$ with Cohen Kappa statistics of 0.904, 0.826 and 0.73 for the three reviewers. Adjudication was required in 20 cases where there was agreement in fewer than five of six diagnostic passes. These included seven biopsies originally diagnosed as simple hyperplasia without atypia, seven as complex hyperplasia without atypia and six as complex hyperplasia with atypia. In all, $65 \%(13 / 20)$ of these cases were diagnosed as EIN at adjudication.

The majority $(78 \%)$ of biopsies originally designated 'complex hyperplasia with atypia' were reclassified as EIN (Table 1). A smaller proportion of lesions designated 'complex hyperplasia without atypia' (44\%) and 'Simple hyperplasia without atypia' (4\%) were reclassified as EIN (Table 1). Of all EIN lesions, 64\% were reclassified from biopsies with 'complex hyperplasia with atypia', $29 \%$ from 'complex hyperplasia without atypia', and $7 \%$ from the simple hyperplasia categories (Figure 1).

Clinical outcome was available in $87 \%(84 / 97)$ of patients based upon hysterectomy results $(n=26$, median interval 68 days), subsequent endometrial sampling with or without additional clinical followup ( $n=46$, median interval 3.5 years) or clinical follow-up alone for a minimum of 1 year $(n=12$, median interval 3.3 years). WHO biopsy diagnoses in these women were distributed among all categories of hyperplasia (Table 2). All eight patients with carcinoma had a prior biopsy classified as EIN. Out of a total of eight cancer outcomes, only one of these EIN cases required adjudication to arrive at an EIN diagnosis (Figure 4, diagnosed according to WHO criteria as nonatypical simple hyperplasia).

Subjective EIN diagnoses stratified well by morphometric $D$-score across a threshold of $D$-score $=1$ (Figure 2). Specifically, all 53 cases with $D$-score $>1$ were judged to be benign (non-EIN) using subjective criteria. Cases with $D$-score $\leq 1$ were more variably interpreted by pathologists, with 27 diagnosed as EIN, and 15 as benign. All future cancer occurrences occurred in patients whose endometrial biopsies were high risk by both objective morphometric $(D$-score $\leq 1)$ and subjective (EIN) methods.

Table 1 Reclassification of WHO hyperplasias using EIN criteria

\begin{tabular}{lcr}
\hline WHO hyperplasia diagnosis & EIN diagnosis n (\%) & Total \\
\hline Complex with atypia & $18(78)$ & 23 \\
Simple with atypia & $0(0)$ & 0 \\
Complex without atypia & $8(44)$ & 18 \\
Simple without atypia & $2(4)$ & 56 \\
Total & $28(29)$ & 97 \\
\hline
\end{tabular}

Differing interpretation of cytology according to WHO hyperplasia and EIN systems is illustrated for two nonatypical hyperplasias diagnosed as EIN (Figures 3 and 4). Both of these cases were followed by endometrial adenocarcinoma.

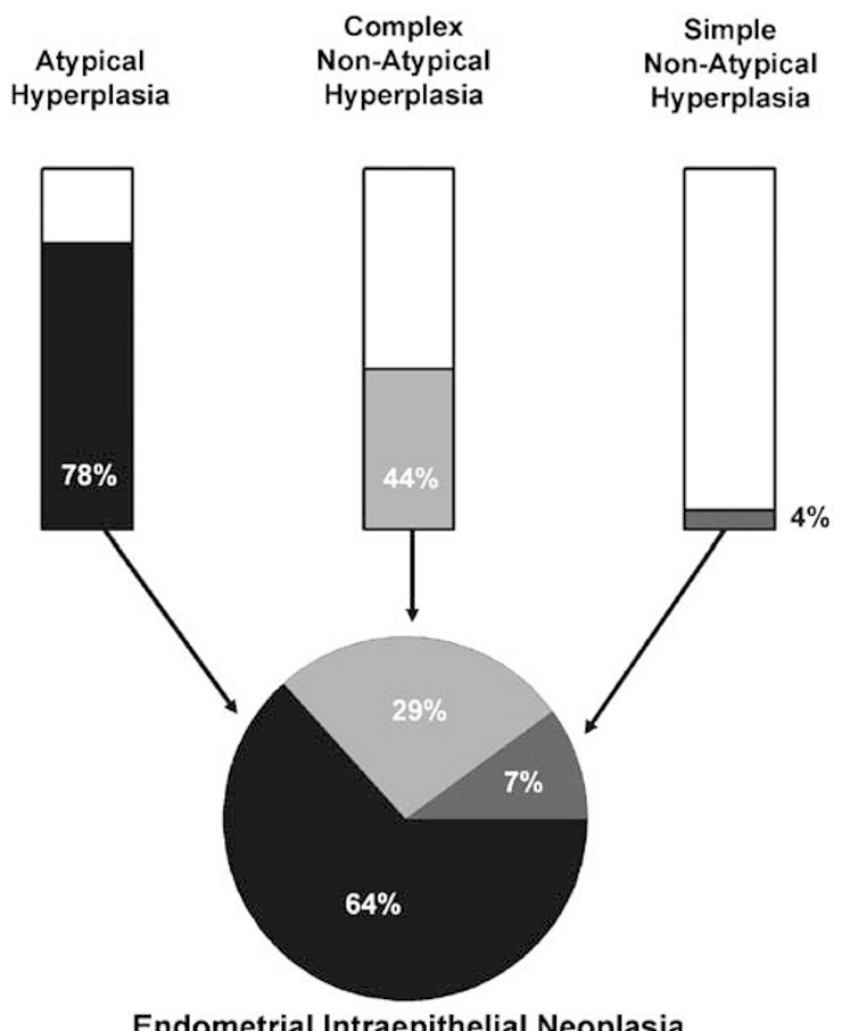

Figure 1 Correlation of WHO and EIN diagnoses. Gray portions of bar graphs show approximate percentages of each WHO hyperplasia class that will be diagnosed as EIN. Remaining WHO hyperplasias not diagnostic of EIN (white) will be allocated to unopposed estrogen (anovulatory), polyp, and other categories. Pie chart shows relative contributions of each hyperplasia type to the EIN diagnostic category in our series of 97 cases with 28 EIN examples.

Table 2 Clinical cancer outcomes by WHO, EIN, and $D$-score

\begin{tabular}{lllc}
\hline Diagnostic schema & Diagnosis & $\begin{array}{r}\text { Cancer, } \\
\mathrm{n}(\%)\end{array}$ & $\begin{array}{c}\text { Total, } \\
\mathrm{n}\end{array}$ \\
\hline WHO hyperplasia & $\begin{array}{l}\text { Complex with atypia } \\
\text { Complex without atypia }\end{array}$ & $\begin{array}{l}5(24) \\
2(13)\end{array}$ & 21 \\
& Simple without atypia & $1(2)$ & 48 \\
& Total & $8(10)$ & 84 \\
& & & \\
& EIN & $8(32)$ & 25 \\
EIN & Non-EIN & $0(0)$ & 59 \\
& Total & $8(10)$ & 84 \\
& & & \\
Morphometry & $D$-score $\leq 1$ (high risk) & $8(21)$ & 38 \\
$(D$-score) & -score $>1$ (low risk) & $0(0)$ & 44 \\
& Total & $8(10)$ & 82 \\
& & & \\
\hline
\end{tabular}



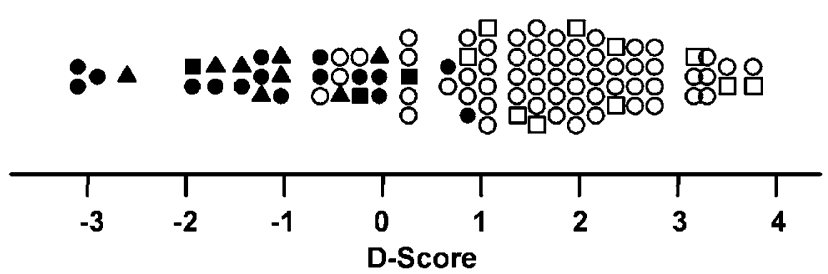

Diagnosis (color)

EIN

Benign

\section{Outcome (symbol) \\ \#an Cancer \\ No Cancer \\ Unknown}

Figure 2 Correlation of subjective EIN diagnosis with objective $D$ score. A $D$-score threshold of 1 , previously established to stratify endometria by cancer risk, ${ }^{7,9-11}$ correlated well with subjective diagnoses of EIN (black, $n=27$ ) compared to benign non-EIN (white, $n=68$ ). All cancer occurrences (triangles) seen during follow-up were captured in the high-risk groups as determined both by subjective (EIN, black) and objective morphometric ( $D$ score $\leq 1$ ) methods.

\section{Discussion}

Identification of endometrial precancers by morphometric $D$-score has proven to be both diagnostically reproducible and predictive of clinical outcome. We show that a subjective implementation of EIN diagnostic criteria is also highly reproducible, consistent with $D$-score predictions, and outperforms the WHO hyperplasia schema in predicting cancer risk.

The reviewed series contained eight adenocarcinoma outcomes. A preceding diagnosis of EIN was $100 \%$ sensitive in predicting all eight endometrial cancers, with $32 \%(8 / 25)$ of EIN diagnoses associated with concurrent or subsequent endometrial adenocarcinoma. This outcome predictive value of a consensus subjective diagnosis was similar to that seen previously for morphometrically diagnosed EIN using the $D$-score as measured by image analysis. ${ }^{11}$ In contrast a biopsy diagnosis of atypical hyperplasia was only $63 \%$ sensitive, predicting five out of eight endometrial adenocarcinoma outcomes. The remaining three cases were initially diagnosed as simple nonatypical ${ }^{1}$ and complex nonatypical ${ }^{2}$ hyperplasias, resulting in all WHO hyperplasia categories being associated with some frequency of cancer, complex atypical (23\%), complex nonatypical $(10 \%)$ and simple nonatypical (2\%) (Table 2 ). Therefore, it is impossible to exclude the possibility of future or concurrent cancer in most patients with a WHO hyperplasia diagnosis of any category.

This latter point highlights a useful aspect of EIN diagnostic scheme that is clinically important in patient management. The very robust negative predictive value of a non-EIN diagnosis (in this study $100 \%$ ) essentially removes most patients from a high-risk category given none of the 59 patients in the non-EIN category were found to have cancer. In contrast, many of these same patients would have

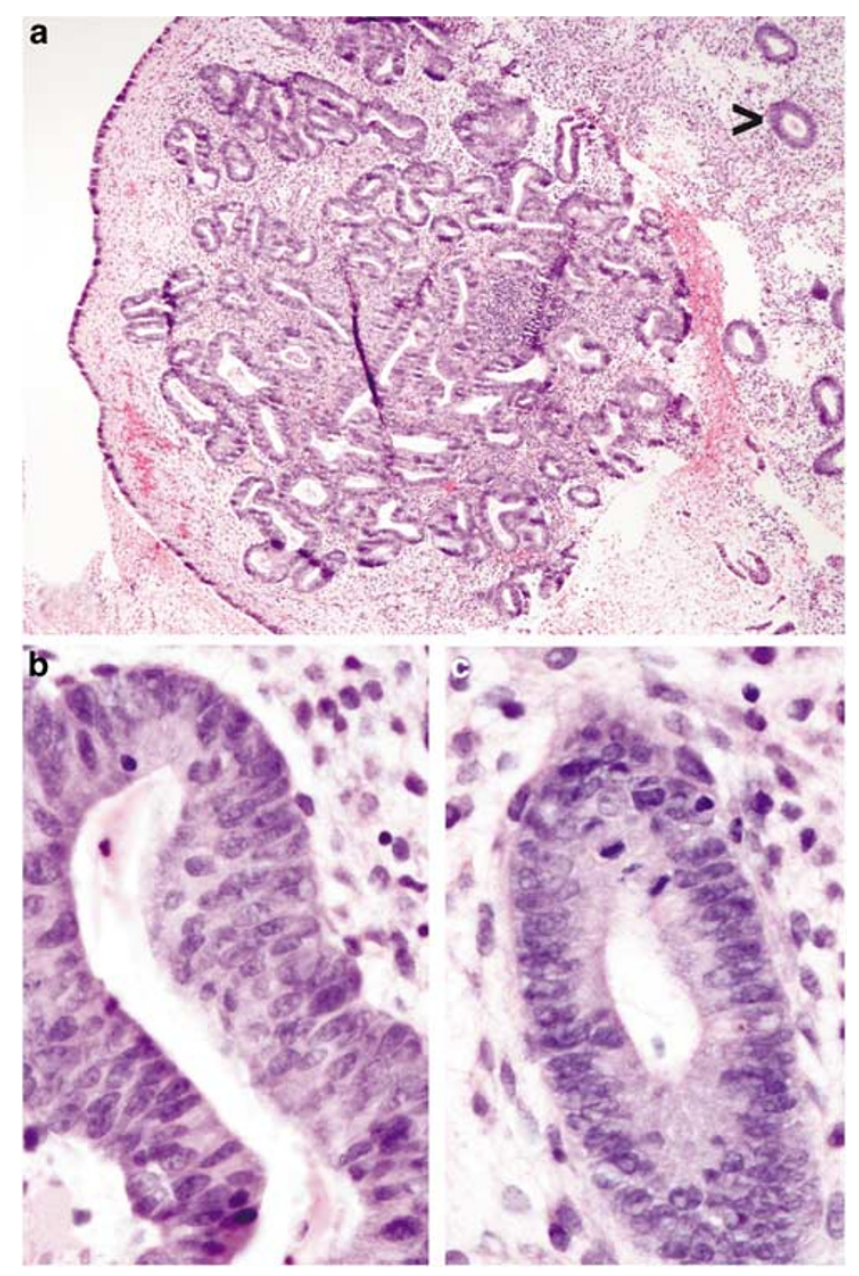

Figure 3 WHO complex nonatypical endometrial hyperplasias reclassified as EIN. A localized area of EIN is seen under low power (panel a) as a jumble of glands whose area exceeds that of intervening stroma, in contrast to loosely packed background proliferative glands (arrowhead). Lesion architecture is a more obvious feature of this EIN that demonstrates subtle cytologic change. Cells of the EIN lesion have larger and more pseudostratified nuclear cytology (panel b) than those of the adjacent proliferative glands (panel c). This 49-year-old patient developed endometrial adenocarcinoma 2 years later as confirmed at hysterectomy.

been needlessly considered to be at increased cancer risk based on WHO classification, for example, 29 of these 59 (16 complex atypical and 13 complex nonatypical hyperplasias) patients would be considered to have at least a $10 \%$ cancer risk. One of the arguments for implementation of the EIN system would be to decrease the frequency of false positive diagnoses, which have significant consequence for the patient.

Interobserver reproducibility of EIN vs non-EIN was good, with all three pathologists agreeing in the first pass in $75 \%$ of cases, and pairwise interobserver kappas of 0.54-0.62. This is better than a recent Gynecologic Oncology Group study where a panel of three gynecologic pathologists agreed on WHO hyperplasia class assignment in only $39 \%$ of cases, 


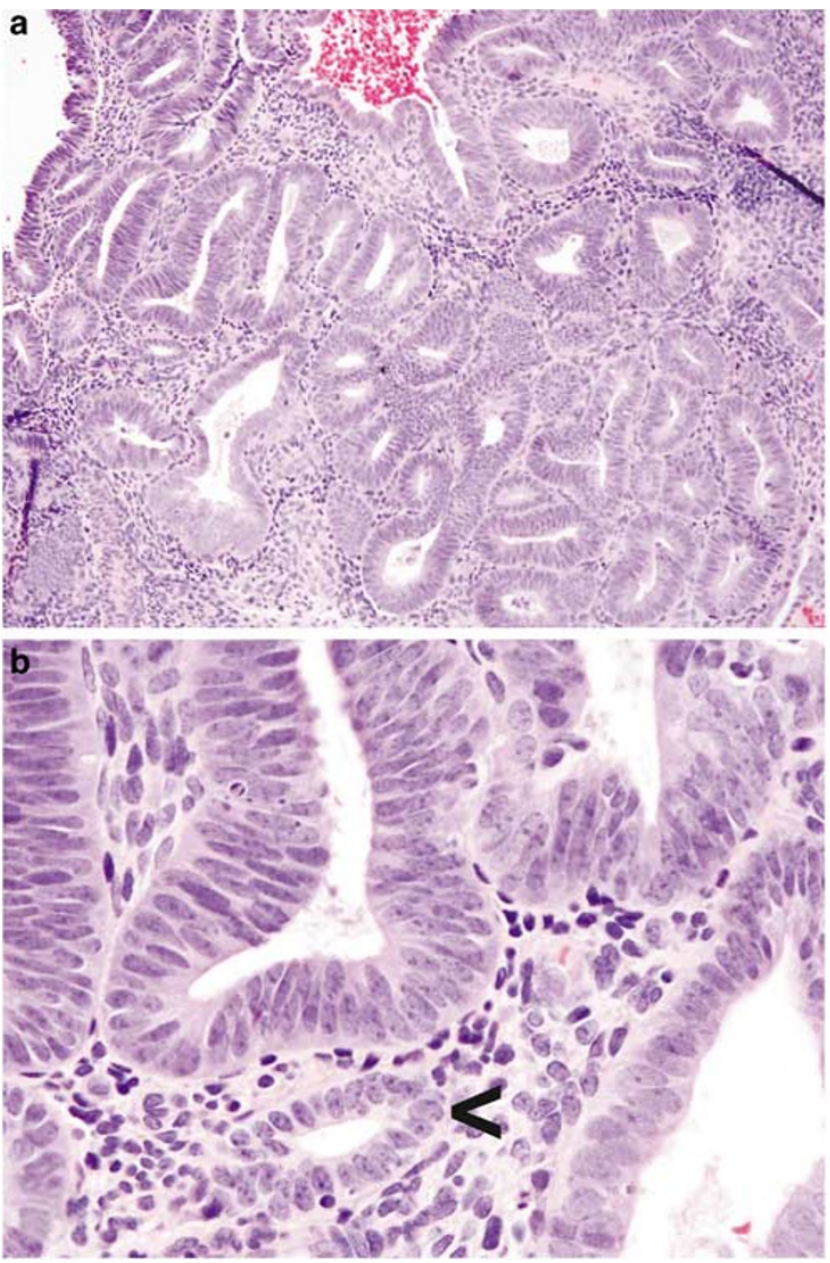

Figure 4 (a) WHO simple nonatypical hyperplasia reclassified as EIN. Monotonous closely packed glands are present throughout the endometrial compartment. The extent of the lesion leaves no broad fields of background for comparison, but isolated 'overrun' normal glands (panel b, arrowhead) demonstrate that in this patient the EIN glands have nuclei that are taller and more polarized than background normal. This 47-year-old patient subsequently developed endometrial adenocarcinoma 8 months later, confirmed at hysterectomy.

with interobserver kappas of $0.34-0.43,{ }^{17}$ and a recent reproducibility study that showed atypical hyperplasia diagnosis interobserver kappa of $0.47 .{ }^{18}$ Improved reproducibility is an expected benefit of contraction of the number of classes to be distinguished. This has been cited by a European group as one justification to contract biopsy diagnosis of premalignant and well-differentiated carcinoma into a single category. ${ }^{19}$ The EIN schema continues to maintain adenocarcinoma as an entity separate from premalignant disease (EIN) because these may be treated differently in the United States, especially when EIN presents in women wishing to maintain fertility. Management of EIN is quite similar to that previously offered to women with an atypical hyperplasia diagnosis, and this may include an option in some cases for hormonal therapy with progestins and careful follow-up surveillance.
While morphometrically low-risk $(D$-score $>1)$ endometria were consistently recognized as nonEIN in our series, morphometrically high-risk endometria ( $D$-score $\leq 1)$, comprised an admixture of subjectively benign and EIN endometria (Figure 2). In this study, we performed morphometry on all intact tissues, irrespective of the presence of endometrial polyps or secretory endometrium that are known to be benign processes that yield 'high risk' $D$-scores. All cases that went on to adenocarcinoma were high risk by both $D$-score $(\leq 1)$ and subjective (EIN) classification, emphasizing the need for the human element in diagnosis.

The most difficult part of EIN diagnosis is exclusion of the many benign mimics that overlap with EIN. Some can be instantly recognized as mimics using features that do not appear in the concise bullet lists of diagnostic criteria. For example, normal secretory endometrium is nonuniform throughout the endometrial thickness. Basal areas without significant stromal predecidual change have much more gland crowding than near the surface where expanding stromal cells push the glands apart. Combined with cytologic differences in secretory activity between the basal and superficial gland elements, it is very easy to misinterpret an isolated fragment of basal secretory endometrium as a localizing EIN lesion. Polyps present another problem, as about $15 \%$ of EIN lesions present within the context of the irregularly distributed glands of a polyp. EIN diagnostic criteria are maintained in a polyp, with the caveat that the polyp itself should be considered the background for comparison of cytology. The polyp context of EIN should always be mentioned in the report, as the entire lesion may be removed in some cases by simple polypectomy.

Education and training are a critical element in achieving diagnostic reproducibility for community deployment of any new diagnostic procedure. To this end, we have developed an online interactive tutorial and deposited online a training series of 50 outcome-annotated endometrial biopsies (the 'EIN Diagnosis Library') at www.endometrium.org. It was this material, coming from patients independent of those studied in the current report, which was used to prepare the least experienced pathologist (JH) to participate in this study. Once learned, EIN diagnoses are robustly applied by pathologists, as indicated by our very good intraobserver reproducibility (kappa 0.73-0.90). Online teaching resources are increasingly popular in many disciplines, but limitations in image resolution and field selection are significant barriers in mimicking the real-time experience of viewing glass slides under the microscope. An example of hardware-induced diagnostic compromise emerged during training for this project. One pathologist had a steady habit of underdiagnosing EIN lesions. Further inquiry revealed that the microscope he was using did not have a $\times 2$ objective, necessary for low-magnification intercomparison of architectural patterns among the many 
tissue fragments scattered throughout the slide. Without this low-power perspective, subtle localizing architectural clues were lost, and the relevant fragments overlooked. When supplied with a $\times 2$ objective, that individual suddenly could quickly recognize the fragment of interest and became concordant with the rest of the group.

The EIN vs benign distinction has been better characterized than that between EIN and cancer. Resolution of EIN from well-differentiated carcinoma relies on histopathologic criteria of solid epithelium, cribriform architecture, mazelike lumens, or myoinvasion. Previous morphometric analysis of endometrial biopsy material from patients with and without myoinvasive cancer on hysterectomy have identified variables such as volume percentage lumen, volume percentage epithelium, and epithelial thickness as features of carcinoma that may predict myoinvasion..$^{20,21}$ These have not yet been extensively tested on new patient series, nor have they been extrapolated to readily applicable subjective criteria. This is a subject that requires more attention, both in terms of developing new molecular markers or criteria for improved diagnostic segregation, and in defining relevant therapeutic thresholds. In particular, there is renewed clinical interest in managing subsets of well-differentiated adenocarcinoma with locally ${ }^{22}$ or systemically delivered hormonal agents. It may become necessary in the near future to critically evaluate new strategies for stratification suited to triage into therapies that are not currently part of our clinical repertoire.

The relationship between EIN and that group of lesions previously diagnosed as endometrial hyperplasia is relevant to practicing pathologists contemplating transition to the EIN diagnosis schema. The canon of 'endometrial hyperplasia,' as defined by the WHO encompasses a biologically diverse assemblage of lesions inconsistently assigned to one of four groups based upon the presence or absence of cytologic atypia and simple or complex architecture. This approach largely derived from a 1985 clinical outcome study of 170 patients in which lesions with cytologic atypia conferred an approximately 14-fold elevated risk for endometrial carcinoma. ${ }^{23}$ Although the WHO 4-class endometrial hyperplasia schema is currently the most widely used classification system for premalignant endometrial lesions, reproducibility of the key assessment of presence or absence of cytologic atypia is poor, with interobserver kappa values of $0.3-0.47 .{ }^{17,18}$ Fixed translation of a specific hyperplasia subtype to EIN category is not possible, because of the poorly defined nature of hyperplasia subgroups, and absence of key EIN criteria such as lesion size and relative standard for cytologic change in the hyperplasia system. Rather than seeking a translation between the systems, EIN criteria must be applied to each case individually in order to achieve the reproducibility and outcome predictive benefits seen in this study.
The EIN diagnostic schema was introduced at Brigham and Women's Hospital in 2002, to replace a local version of the older hyperplasia-based nomenclature. EIN implementation has been very well received by our clinical colleagues, who were primed by a series of explanatory conferences and memos in advance of a go-live date. The ease of pairing each diagnostic entity with clinical management facilitates clear communication. It has, however, raised new questions. Now that we are increasingly conscious of lesion size and extent while making the diagnosis, there is interest in exploration of whether further subdivision by any of these variables (other than the simple $1 \mathrm{~mm}$ size threshold) has any clinical meaning. Further studies are needed to resolve these issues.

\section{Acknowledgements}

This work was supported in part by NIH Grant RO1CA92301 (G Mutter).

\section{References}

1 Bokhman J. Two pathogenetic types of endometrial carcinoma. Gynecol Oncol 1983;15:10-17.

2 Sherman ME, Sturgeon S, Brinton L, et al. Endometrial cancer chemoprevention: implications of diverse pathways of carcinogenesis. J Cell Biochem 1995;59(Suppl 23):160-164.

3 Mutter GL, The Endometrial Collaborative Group. Endometrial intraepithelial neoplasia (EIN): will it bring order to chaos? Gynecol Oncol 2000;76:287-290.

4 Silverberg SG, Mutter GL, Kurman RJ, et al. Tumors of the uterine corpus: epithelial tumors and related lesions. In: Tavassoli FA, Stratton MR (eds). WHO Classification of Tumors: Pathology and Genetics of Tumors of the Breast and Female Genital Organs, 1st edn. IARC Press: Lyon, France, 2003, pp 221-232.

5 Mutter GL, Baak JPA, Crum CP, et al. Endometrial precancer diagnosis by histopathology, clonal analysis, and computerized morphometry. J Pathol 2000;190: 462-469.

6 Mutter GL, Lin MC, Fitzgerald JT, et al. Altered PTEN expression as a diagnostic marker for the earliest endometrial precancers. J Natl Cancer Inst 2000;92: 924-930.

7 Baak JPA, Nauta J, Wisse-Brekelmans E, et al. Architectural and nuclear morphometrical features together are more important prognosticators in endometrial hyperplasias than nuclear morphometrical features alone. J Pathol 1988;154:335-341.

8 Baak JP, Wisse-Brekelmans EC, Fleege JC, et al. Assessment of the risk on endometrial cancer in hyperplasia, by means of morphological and morphometrical features. Pathol Res Pract 1992;188:856-859.

9 Dunton C, Baak J, Palazzo J, et al. Use of computerized morphometric analyses of endometrial hyperplasias in the prediction of coexistent cancer. Am J Obstet Gynecol 1996;174:1518-1521.

10 Orbo A, Baak JP, Kleivan I, et al. Computerised morphometrical analysis in endometrial hyperplasia 
for the prediction of cancer development. A long-term retrospective study from northern Norway. J Clin Pathol 2000;53:697-703.

11 Baak JP, Orbo A, van Diest PJ, et al. Prospective multicenter evaluation of the morphometric $D$-score for prediction of the outcome of endometrial hyperplasias. Am J Surg Pathol 2001;25:930-935.

12 Orbo A, Baak JP. Computer-based morphometric image analysis of endometrial hyperplasia. Tidsskr Nor Laegeforen 2000;120:496-499.

13 Mutter GL. Diagnosis of premalignant endometrial disease. J Clin Pathol 2002;55:326-331.

14 Mutter GL, EIN Central [On Line]. http://www.endometrium.org, 2001.

15 Ronnett BM, Kurman RJ. Precursor lesions of endometrial carcinoma. In: Kurman R (ed) Blaustein's Pathology of the Female Genital Tract, 5th edn. Springer-Verlag: New York, 2002, pp 467-500.

16 Mutter GL. Histopathology of genetically defined endometrial precancers. Int J Gynecol Pathol 2000;19: 301-309.

17 Zaino R, Trimble C, Silverberg S, et al. Reproducibility of the diagnosis of atypical endometrial hyperplasia (AEH): a gynecologic oncology group (GOG) study. Lab Invest 2004;84(Suppl 1):218A.
18 Kendall BS, Ronnett BM, Isacson C, et al. Reproducibility of the diagnosis of endometrial hyperplasia, atypical hyperplasia, and well-differentiated carcinoma. Am J Surg Pathol 1998;22: 1012-1019.

19 Bergeron C, Nogales F, Masseroli M, et al. A multicentric European study testing the reproducibility of the WHO classification of endometrial hyperplasia with a proposal of a simplified working classification for biopsy and curettage specimens. Am J Surg Pathol 1999;23:1102-1108.

20 Baak JPA, Kurver P, Overdiep S, et al. Quantitative, microscopical, computer-aided diagnosis of endometrial hyperplasia or carcinoma in individual patients. Histopathology 1981;5:689-695.

21 Baak JPA, Kurver PHJ, Boon ME. Computer-aided application of quantitative microscopy in diagnostic pathology. Pathol Annu 1982;17:287-306.

22 Montz FJ, Bristow RE, Bovicelli A, et al. Intrauterine progesterone treatment of early endometrial cancer. Am J Obstet Gynecol 2002;186:651-657.

23 Kurman R, Kaminski P, Norris H. The behavior of endometrial hyperplasia: a long term study of 'untreated' hyperplasia in 170 patients. Cancer 1985; 56:403-412. 